\title{
Web-based neuromuscular simulator applied to the teaching of principles of neuroscience
}

\author{
Leonardo Abdala Elias*, André Fabio Kohn
}

Abstract Introduction: The learning of core concepts in neuroscience can be reinforced by a hands-on approach, either experimental or computer-based. In this work, we present a web-based multi-scale neuromuscular simulator that is being used as a teaching aid in a campus-wide course on the Principles of Neuroscience. Methods: The simulator has several built-in individual models based on cat and human biophysics, which are interconnected to represent part of the neuromuscular system that controls leg muscles. Examples of such elements are i) single neurons, representing either motor neurons or interneurons mediating reciprocal, recurrent and Ib inhibition; ii) afferent fibers that can be stimulated to generate spinal reflexes; iii) muscle unit models, generating force and electromyogram; and iv) stochastic inputs, representing the descending volitional motor drive. Results: Several application examples are provided in the present report, ranging from studies of individual neuron responses to the collective action of many motor units controlling muscle force generation. A subset of them was included in an optional homework assignment for Neuroscience and Biomedical Engineering graduate students enrolled in the course cited above at our University. Almost all students rated the simulator as a good or an excellent learning tool, and approximately $90 \%$ declared that they would use the simulator in future projects. Conclusion: The results allow us to conclude that multi-scale neuromuscular simulator is an effective teaching tool. Special features of this free teaching resource are its direct usability from any browser (http://remoto.leb.usp.br/), its user-friendly graphical user interface (GUI) and the preset demonstrations.

Keywords Biomedical engineering education, Biological system modeling, Computational biology, Neural engineering. 


\section{Introduction}

Computational tools have been extensively adopted in undergraduate and graduate courses from different fields to the teaching and learning of fundamental concepts (e.g., (Dolecek, 2012; Illert et al., 1992; McGrath et al., 2003; Touretzky et al., 2003)). In the fields of neurosciences and motor control, the learning of the information provided in the classroom or in textbooks may be improved by a hands-on approach, either using wet lab experiments or computer simulations. Currently, ethical issues have prevented the wide use of animals for generalpurpose experimentation. Besides, experiments (even simple ones) may require highly specialized equipment and devices, which are frequently available in research labs only. At the same time, biologically plausible mathematical models have become largely accessible by means of public databases (Morse, 2008) including those that are able to represent with fidelity several aspects of the nervous and musculoskeletal systems. This, allied to the increased power of personal computers, allows the student to reinforce his/her learning through active computerbased experimentation.

General-purpose simulators have been developed either with a focus on computational neuroscience or on motor control/biomechanics (e.g., (Bower and Beeman, 1998; Brette et al., 2007; Carnevale and Hines, 2006; Delp et al., 2007)). These packages can also be used for teaching or learning purposes; however, both the student and the instructor must have some familiarity with programming languages and a reasonable effort is needed to develop the structure to be studied. Moreover, the integration between different subsystems (primarily, the link between the nervous and the musculoskeletal systems) may be an arduous task, which may divert the instructor and student from the main focus.

Different multi-scale models have already been developed for the study of the neuromuscular system (Bashor, 1998; Fuglevand et al., 1993; Raphael et al., 2010; Winters, 1995). Nonetheless, these models were mainly intended to be used as tools for research labs and frequently their source-codes are not readily available or they are platform-dependent, limiting their applicability as educational tools. The largescale neuromuscular system model developed by Maw et al. (1996) was primarily intended for education; however, the source code is not available and it is platform-dependent. Conversely, the simulator for neural networks described in (Ziv et al., 1994) is being used for research and educational purposes, but it's focus is only on neural mechanisms. Therefore, it does not have the necessary tools for the student to understand how force is developed and how it depends jointly on the properties of the muscle fibers and the nervous system.

In this report, a specific-purpose neuromuscular simulator will be presented and its use as a tool for the teaching of Principles of Neuroscience will be described. This web-based simulator, named ReMoto, provides several tools that are very useful for the study of purely neurophysiological concepts, from single cells to neuronal networks. In addition, due to the inclusion of some aspects of muscle unit physiology, it may also be used to explore the intricacies of the generation of muscle force and muscle electrical activity. The first version of this neuromuscular system was intended for computational neuroscience research (Cisi and Kohn, 2008). The recently redesigned graphical user interface (GUI) and the inclusion of novel experimental protocols (called "Demonstrations" in the simulator) opened the way for its use for educational purposes. Several application examples will be provided after the presentation of the main details of the simulator, ranging from single cell behavior to motor behavior. Finally, the outcomes of a survey conducted on students from the Principles of Neuroscience course (see Course description in Methods) will be presented. Part of this work was previously presented as an abstract (Elias et al., 2012b).

\section{Methods}

\section{Course description}

The Principles of Neuroscience course is offered at the graduate entry level for students coming from different backgrounds (e.g., engineers, physical therapists, biologists). The course is officially offered in both the Electrical/Biomedical Engineering Graduate Program and in the campus-wide Neuroscience Graduate Program at the University de São Paulo (USP, São Paulo, Brazil). The topics covered include: structure of the nervous system, structure of nerve cells and their membranes, ionic channels in the neuronal membrane, the ionic basis of the resting potential and the action potential, presynaptic and postsynaptic mechanisms in synaptic function, LTP and synaptic plasticity in memory and learning, sensory receptors in the skin and muscles, muscle contraction mechanisms, basics of spinal cord neural circuits and their influence on the control of muscles acting around a joint, the inner ear (vestibular and cochlear functions), electrical activity of the cortex and sleep.

The classes are based on an active interplay between the instructor (second author of this study) and 
the students. They are required to come to class with a pre-specified topic of the textbook well studied at home. The instructor pinpoints the most important concepts of that lecture and answers the questions posed by the students. At the end of randomly selected classes, there is an essay-based mini-exam. Therefore, the students come to every class with the subject matter well read beforehand. At the end of the course there is a final exam. There are two optional computer simulation home-works, one based on the Hodgkin-Huxley model (Hodgkin and Huxley, 1952; Touretzky et al., 2003) and the other based on ReMoto. A student's performance in these home-works contributes with a small percentage to the final grade.

\section{Description of the neuromuscular model}

\section{General structure}

A complete description of the mathematical models implemented in the simulator may be found elsewhere (Cisi and Kohn, 2008; Elias et al., 2012a). Briefly, the simulator provides a detailed modeling of four spinal motor nuclei that command leg muscles responsible for ankle extension (Soleus - SOL; Gastrocnemius Medialis - GM; Gastrocnemius Lateralis - GL) and ankle flexion (Tibialis Anterior - TA). Each nucleus encompasses a motor neuron (MN) pool and spinal interneurons (INs) mediating recurrent inhibition (Renshaw cells - RC), reciprocal inhibition (Ia inhibitory INs that receive inputs from antagonist muscles), and $\mathrm{Ib}$ inhibition. Ia and $\mathrm{Ib}$ afferents are present in ReMoto to allow studies of spinal reflexes (e.g., H-reflex) generated by electrical stimulation applied to a nerve (Posterior Tibial Nerve for SOL, GL and GM; Common Peroneal Nerve for TA). This feature of nerve stimulation is useful for the understanding of some electrophysiological tests applied in the neurology clinic. Default numbers of spinal neurons and afferent axons were based on estimates from human and cat literature, but these values can be changed according to the application being studied (see examples in Results section). The MN pool drives muscle units, which generate both electrical (motor unit action potentials - MUAPs) and mechanical activity (force twitches). For each muscle, one output is the EMG, expressed as the sum of all MUAPs, and the force, which is the sum of all muscle twitches. Figure 1 summarizes the network structure of two motor nuclei (SOL and TA) and the pathways mediating monosynaptic Ia excitation, Ib inhibition, recurrent inhibition, and reciprocal inhibition. It is interesting to note that these modeled neural circuits may influence each other by means of the well-known reciprocal inhibitory pathways.
Each type-specified (i.e., S, FR and FF) MN model is represented as two cylindrical compartments associated, respectively, with the soma and the dendritic tree. The somatic compartment encompasses $\mathrm{Na}^{+}$and fast $\mathrm{K}^{+}$conductances, responsible for the genesis of action potentials, and a slow $\mathrm{K}^{+}$conductance yielding the afterhyperpolarization (AHP). Recently, an L-type $\mathrm{Ca}^{++}$channel was added to the dendritic compartment to account for the persistent inward current (PIC), which is activated by the presence of neuromodulators (e.g., serotonin and norepinephrine) in the spinal cord (Elias and Kohn, 2013; Heckman et al., 2009). The level of neuromodulation can be adjusted to set the PIC magnitude during the simulation of different motor tasks (Elias and Kohn, 2013; Elias et al., 2012a). In addition, synaptically activated conductances (excitatory and inhibitory) are included in both somatic and dendritic compartments. INs are modeled as a single compartment (point models) with conductances similar to those included in the MN soma, but without PIC-generating conductances. The dynamic behavior of each conductance is a simplification of the HodgkinHuxley formalism (Destexhe, 1997) that was adopted to speed up the simulations. Parameter values were chosen so that individual neuron models matched experimental data from cats (e.g., AHP time course; firing rate versus injected current relation). The synaptic mechanisms are modeled following a kinetic model (Destexhe et al., 1994) with facilitation and depression dynamics, i.e., the amplitude of post-synaptic potentials can increase or decrease, respectively, after repetitive activations. A spike generated by a MN model reaches the MU end plate after a randomly chosen delay value, representing the effect of axonal conduction velocity. Similarly, there are delays in synaptic transmission from descending commands and afferent fibers to spinal neurons.

The MUAPs are modeled by Hermite-Rodrigez functions (Lo Conte et al., 1994; Zhou and Rymer, 2004), which are summed to generate the EMG. A band-pass filter is applied to the signal in order to reproduce in the simulator what happens in a real bipolar surface EMG acquisition. The MU twitch is modeled as the impulse response of a critically damped second-order system which approximates the behavior obtained from human muscles (MilnerBrown et al., 1973).

In ReMoto, two nerves that supply the Triceps Surae (SOL, GM, and GL) and the TA can be electrically stimulated (short current pulses) as done in clinical neurophysiology, to obtain M-waves and H-reflexes. These electrical response signals are useful for studying the human spinal cord and nerves by noninvasive means, either in health or disease 


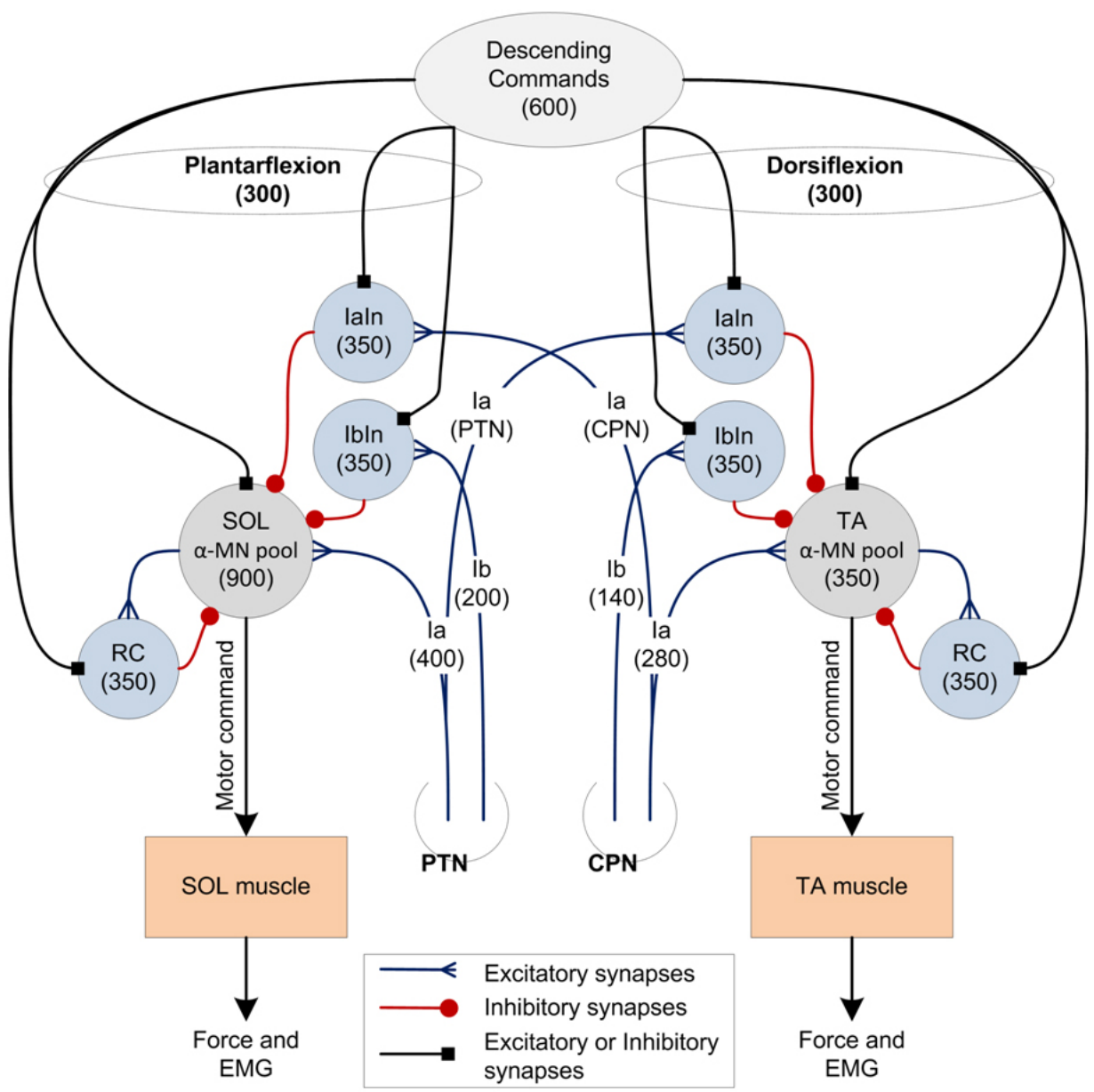

Figure 1. Schematic of the simulator. This figure shows only two of the motor nuclei, one for the Soleus (SOL) and the other for the Tibialis Anterior (TA) muscle. The descending commands, responsible for ankle extension and/or ankle flexion, activate the $\alpha$-MN pools and spinal neurons: Renshaw cells (RCs), Ia inhibitory interneurons (IaIn), and Ib inhibitory interneurons (IbIn). Values in parentheses show default values of the numbers of elements. Ia and Ib afferents come from Posterior Tibial Nerve (PTN) and Common Peroneal Nerve (CPN), which can be electrically stimulated.

(Pierrot-Deseilligny and Burke, 2012). In addition, electrical stimuli may be intracellularly applied to spinal neurons with different waveforms (step, pulse, sinusoid, ramp, and random signal) for studies of single neuron dynamics as done in invasive animal experiments. In order to mimic volitional force control, the MN pool may be driven by descending tracts whose spike trains are modeled as nonhomogeneous renewal point processes with Gamma-distributed interspike intervals, which provide a flexible and realistic stochastic source for the spinal neurons (Deger et al., 2012). Point process intensities may be modulated by different mathematical functions (e.g., sinusoid) in order to reproduce dynamic motor behaviors.

The simulator was developed in Java ${ }^{\circledR}$ (Oracle) programming language, as a web application (accessible at http://remoto.leb.usp.br/). It uses open source technologies, which include $i$ ) Eclipse as the development environment ii) Tomcat as the servlet container iii) HSQLDB as the database manager system iv) Struts as the web framework $v$ ) and JFreeChart as the chart library. ReMoto is a multi-platform application that can be accessed from any web browser with support to the Java ${ }^{\circledR}$ virtual machine, including those embedded in mobile devices (e.g., smartphones and tablets). The user can also work in a logged-in mode in which he/she could create specific simulation scenarios that remain available for his/her future studies. A series of default scenarios are provided to assist first-time users as well as instructors.

\section{Graphical user interface (GUI)}

The GUI was greatly improved in the new version of the simulator in comparison with the previous 
one, which was mainly intended for computational neuroscience research (see Figure 2 in (Cisi and Kohn, 2008)). The new GUI is designed with interactive menus representing more general structures (e.g., number of neuronal elements as shown in Figure 2A) and internal menus permitting access to more specific elements. In addition, the user can now visualize the selected input waveforms and/or the statistical characteristics of these signals when setting up the simulation configuration (see Figure 2B), improving the control over what one wants to simulate. All these new features increased the potential use of ReMoto as a teaching tool.

\section{Visualizing and exporting output data}

Several output data can be visualized directly from the simulation environment (see Figure 2C). An interesting additional feature of the most recent version is the possibility to visualize the output data in an interactive graph (by means of an Applet, which is a Java ${ }^{\circledR}$ application embedded in the HTML code), with zooming and dragging capabilities. However, if the user wants to perform a more specific analysis of the simulated data (e.g., peri-stimulus time histograms, EMG envelope, etc.) he/she may export the data in ASCII format, which is readable by most math programs (e.g., MatLab ${ }^{\circledR}$ ) or spreadsheets (e.g., MS Excel ${ }^{\circledR}$ ).

\section{Results}

\section{Application examples}

\section{Single neuron responses: action potential, synaptic facilitation, refractory period, and frequency-current relationship}

The first set of examples will show how the neuromuscular simulator can be used to illustrate basic behaviors of a single neuron in response to different stimuli. Figure 3 A shows simulation results from a single neuron (S-type MN from the SOL motor nucleus), which received synaptic inputs from Ia afferents of the PTN. Five electrical pulses stimulated this nerve so that the presynaptic input generated excitatory post-synaptic potentials (EPSPs) on the target neuron. The temporal summation of these EPSPs leads to membrane potential depolarization up to a given level (threshold) from which the action potential (AP) is generated by the ionic channel dynamics (see the inset in Figure 3A). The rising phase (or depolarization) of the AP occurs due to the opening of $\mathrm{Na}^{+}$channels while its falling phase (or repolarization) is due to the opening of $\mathrm{K}^{+}$channels, as well as the closing of $\mathrm{Na}^{+}$channels (inset of Figure $3 \mathrm{~A}$ ). This is the basic mechanism of AP generation that was firstly described in the seminal paper by Hodgkin and Huxley (1952). However, in a large variety of neurons (e.g., MNs) one can observe a hyperpolarization following the AP (referred to as the AHP), which is mainly due to a slow $\mathrm{K}^{+}$channel, and is functionally significant in regulating the neuronal firing rate during a sustained activity (Kernell, 2006). All these mechanisms can be easily explored using the simulator, and, in addition to the somatic membrane potential, one can monitor the dendritic membrane potential, which is the main location for synaptic inputs (see the red curve in Figure 3A).

A quite interesting topic covered in Neuroscience courses is the synaptic plasticity (Kandel et al., 2013). A kind of short-term synaptic plasticity is synaptic facilitation, which is an increase in EPSP amplitude during repetitive presynaptic activity. The main cause of this phenomenon is an excess (or residue) of $\mathrm{Ca}^{++}$ ions within the presynaptic membrane, leading to an increase in neurotransmitter release. Using the simulator, one can investigate how the synaptic facilitation time course (which may be easily changed) influences the genesis of an AP. Figure 3B shows an example of the effect of synaptic facilitation on the genesis of an AP. As the EPSP amplitude increases after each presynaptic spike, the AP threshold is reached earlier. The student/instructor can also simulate the opposite effect, i.e., synaptic depression (not shown here) that occurs during long-lasting presynaptic activity (Kandel et al., 2013; Kohn et al., 1997).

After the genesis of an AP, a second spike can only be generated after a certain minimal time interval referred to as the refractory period $\left(T_{\mathrm{r}}\right)$. This lowexcitability period of the neuron is due to the AHP and to the $\mathrm{Na}^{+}$channel inactivation (Kandel et al., 2013). The double pulse experiment illustrated in Figure 4 is frequently used to evaluate this property. The first stimulus pulse is injected into the neuron's soma so as to generate an AP. The second pulse is injected after a delay and having an amplitude which is barely supra-threshold. The minimal delay value that results in the firing of an AP by the second stimulus pulse defines $T_{\mathrm{r}}$ (see the small blue and red bars in Figure 4 indicating the two stimulus pulses that were able of firing the neuron once per pulse). If the delay between the two injected pulses is lower than $T_{\mathrm{r}}$ an AP cannot be generated (see the black vertical bar in Figure 4), unless the amplitude of the current is increased (relative refractory period). Within the absolute refractory period, which is primarily due to the $\mathrm{Na}^{+}$channel inactivation, no AP will be generated even for high intensity pulses (not shown). 

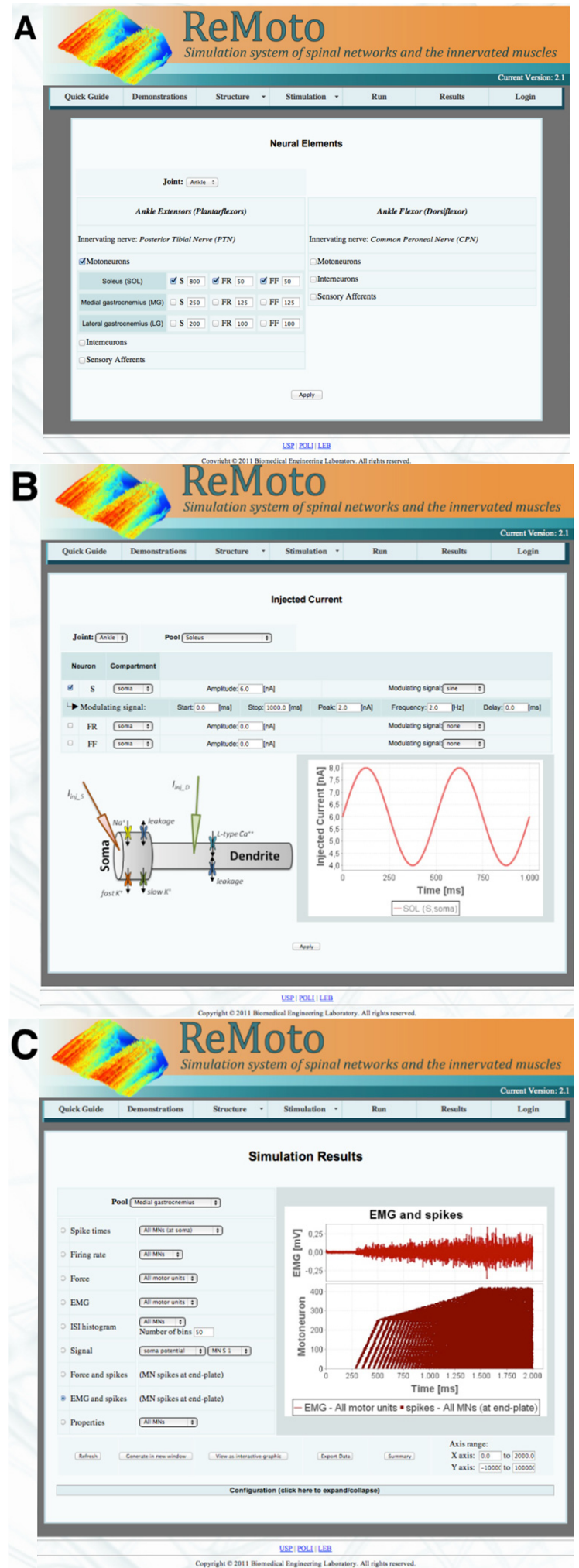

Figure 2. Screenshots taken from different parts of ReMoto. The graphical user interface (GUI) was entirely redesigned in comparison with the first version of the simulator (Cisi and Kohn, 2008). (A) Selection of neuronal elements: In this screen, the user can select the elements that will be used in a specific simulation. (B) Configuration of the injected current: The graph illustrates the current waveform that is injected into the neuron's soma (a DC level plus a sine wave). (C) In the Simulation Results page, several output variables can be visualized and, as additional tools, the user can visualize the data in an interactive graph as well as export the data in ASCII format. 

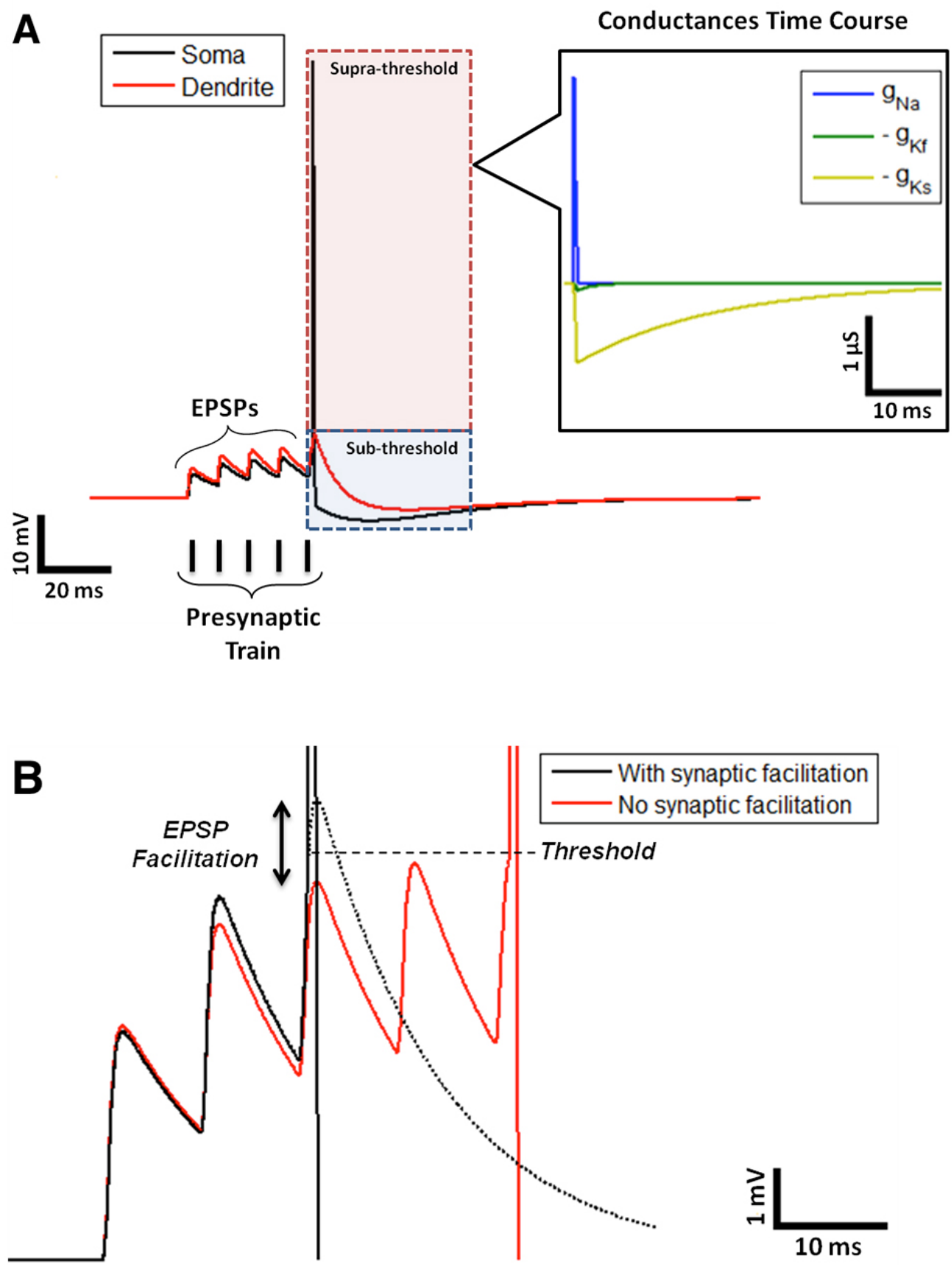

Figure 3. (A) Temporal summation of excitatory post-synaptic potentials (EPSPs) leading to action potential (AP) generation when the somatic membrane crosses a firing threshold. The AP firing depends on the ionic conductances activated (inset). Depolarization depends on the $\mathrm{Na}^{+}$conductance $\left(g_{\mathrm{Na}}\right)$ and repolarization on both $g_{\mathrm{Na}}$ and fast $\mathrm{K}^{+}\left(g_{\mathrm{Kf}}\right)$. A slow K $\mathrm{K}^{+}$conductance $\left(g_{\mathrm{Ks}}\right)$ yields the afterhyperpolarization (AHP). All these variables are readily available as simulation outputs. (B) Somatic membrane time courses in two conditions: without and with synaptic facilitation. Note short-term increases in EPSP amplitudes during facilitation, which makes the membrane potential reach threshold earlier, leading to AP firing (most of it is clipped for clearer viewing of the EPSPs).

A final example concerning the basic mechanisms of single neurons is the response to steady currents injected through the cell's membrane. Figure 5A shows qualitatively that the higher is the amplitude of the injected current the higher is the neuron's firing rate (APs per seconds). This is valid for a great variety of neurons in the nervous system and the instructor may point out that many sensory receptors have their 


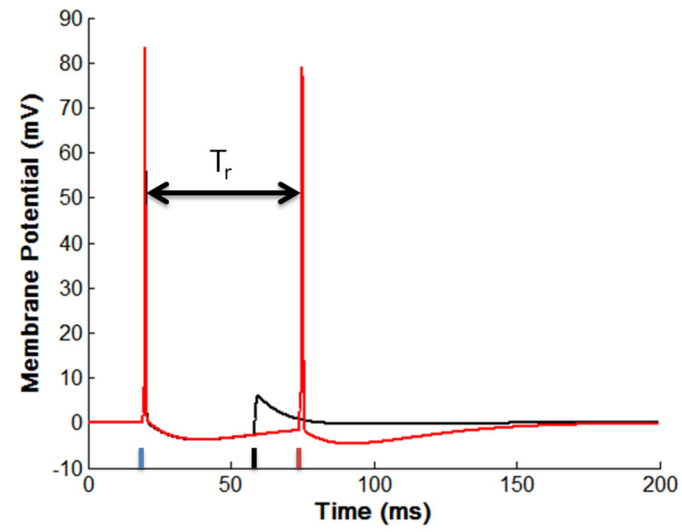

Figure 4. Double pulse paradigm used to evaluate the refractory period of a neuron. When the interpulse interval (IPI) is about or higher than $55 \mathrm{~ms}$ (small gray vertical bars at the bottom) the neuron discharges a second spike; however, for lower IPIs the second pulse (barely supra-threshold) does not generate a spike (see EPSP following the black vertical bar) due to the refractory period of the neuron.

functioning based on a monotonic relation between firing rate and input intensity, as shown in Figure 5B. For more advanced students, the instructor may point out that in some cases the relation between the neuron's firing rate $(f)$ and the injected current $(I)$ - referred to as $f-I$ curve - may be approximately linear (Figure 5B), as in anaesthetized cats (Kernell, 2006), and at other times it will be nonlinear as in decerebrate non-anaesthetized cats or in different animals after administration of neuromodulators (e.g., serotonin or norepinephrine) (Elias and Kohn, 2013; Kernell, 2006)).

\section{Frequency modulation and phase locking}

This application, illustrated using a single neuron, is more challenging since it evidences the highly nonlinear behavior of the neuron as an informationprocessing unit. Initially, a steady current is injected into the neuron so as to maintain an approximately constant firing rate $(\sim 20 \mathrm{~Hz})$. Next, a sinusoidal modulation is superimposed to the steady current and will be called simply the input to the neuron.

For very low input frequencies ( $2 \mathrm{~Hz}$ in Figure 6A) the neuron's firing rate is sinusoidally modulated, i.e., the output rate follows the input signal. This relation between the neuron's output firing rate and the neuron's input amplitude is the so-called rate coding principle of sensory neurophysiology (Kandel et al., 2013). As the input frequency increases, a nonlinear behavior starts to emerge. For instance, for a $6 \mathrm{~Hz}$ input frequency (Figure 6B) the neuron output synchronizes with the input in a 3:1 fashion, i.e., the neuron discharges three APs at every cycle of the sinusoidal input. It is worth noting that the discharge burst occurs at the
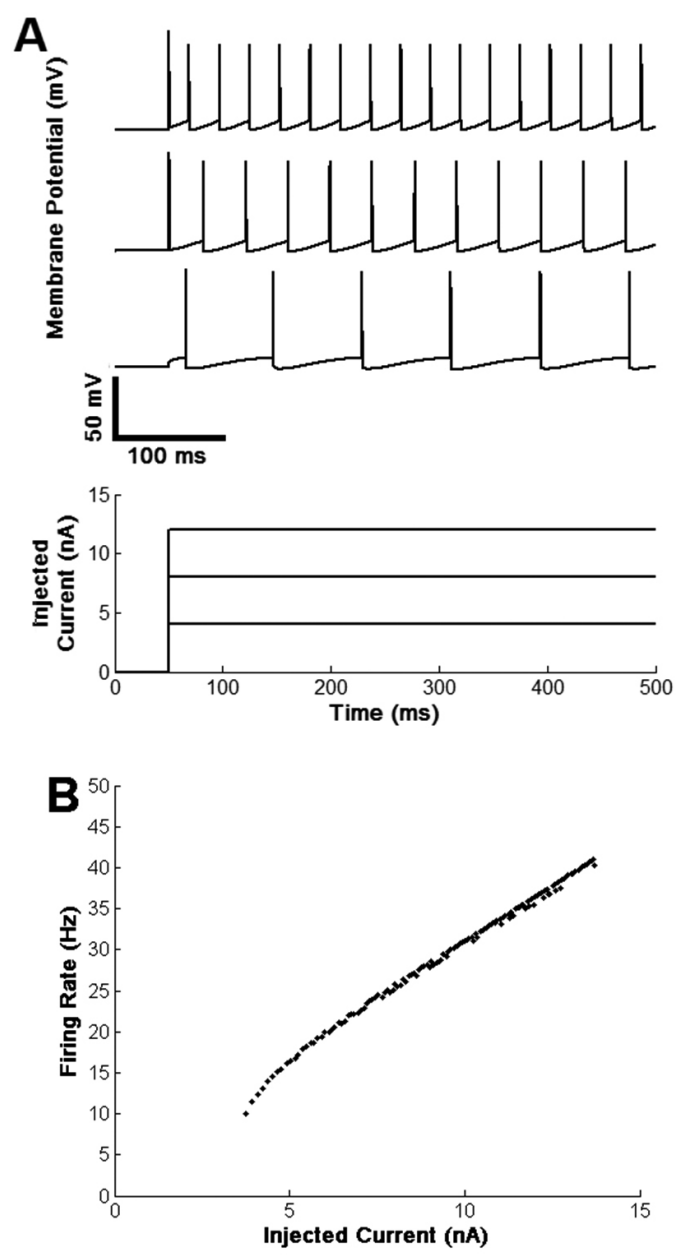

Figure 5. (A) A steady current injected into the neuron's soma evokes repetitive discharges with a certain frequency (firing rate). The higher the injected current amplitude, the higher the neuron's firing rate. (B) Relationship between injected current and firing rate for a single motor neuron model. This approximately linear relationship is characteristic for motor neurons of anaesthetized animals and for several other neurons within the central nervous system (CNS).

same phase of the sinusoid, characterizing the phase locking phenomenon. For modulation frequencies in a range around the natural frequency of the neuron (i.e., $20 \mathrm{~Hz}$ ), a 1:1 synchronism is observed (note that one AP is generated at each cycle of the sinusoid in Figure 6C). Finally, for frequencies well above the natural frequency, a 1:M (with $M$ representing the number of input cycles) synchronism is observed. For example, if the modulation frequency is set at $100 \mathrm{~Hz}$ (Figure 6D) for the neuron used in this application, a 1:4 synchronism is achieved. This intricate phenomenon, whose mechanisms are out of the scope of this paper, has been described in many neural systems, such as in invertebrate sensory neurons (Martinez et al., 1983) and neurons of the auditory system (cochlear nerve 
A

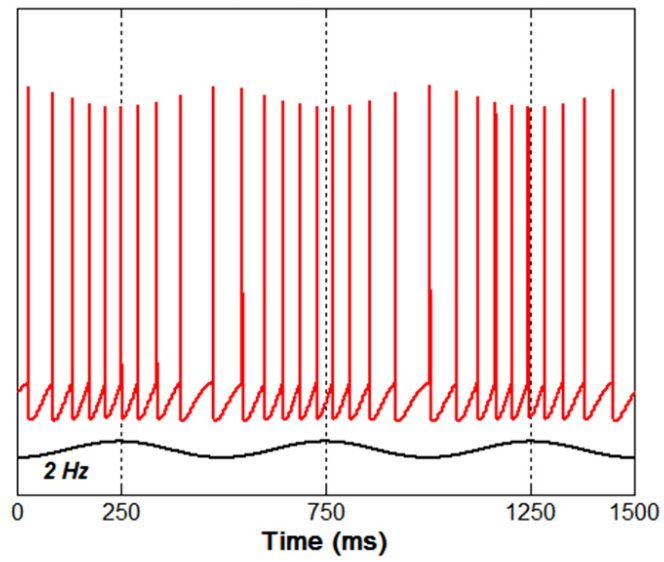

C

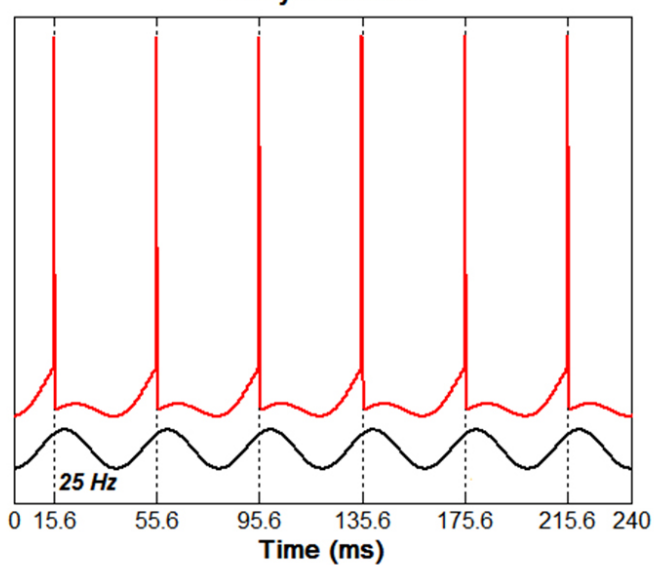

B

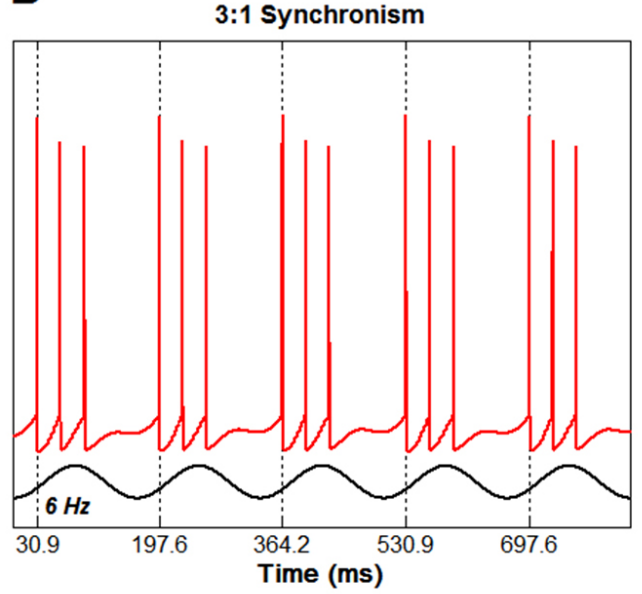

D

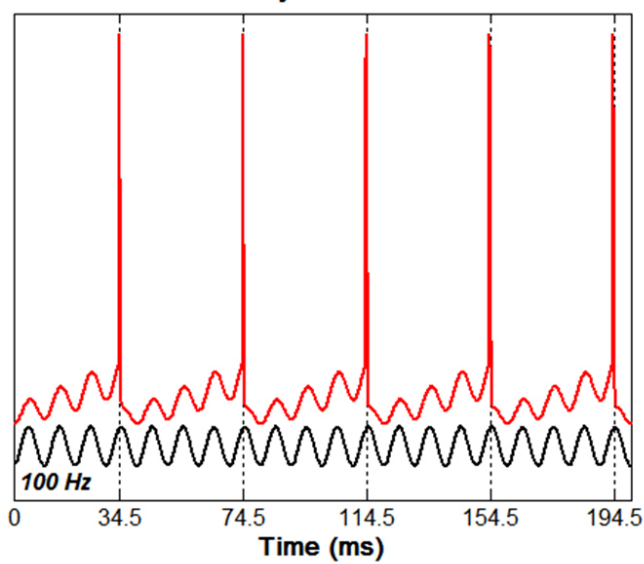

Figure 6. Frequency modulation and phase locking between the input and output of a single neuron model. (A) For a low-frequency sinusoidal input, the neuron's firing rate approximately follows the input signal imposed on the neuron. (B) 3:1 synchronism is shown when the input frequency is set to $6 \mathrm{~Hz}$. This frequency is well below the natural frequency of this neuron $(f=20 \mathrm{~Hz})$. Note that the triple discharge occurs at the same phase in each cycle of the sinusoidal input. (C) When the input frequency is near $f_{\mathrm{n}}$, a $1: 1$ synchronism is observed. (D) For an input at $100 \mathrm{~Hz}, 1$ spike is discharged at every 4 cycles of the input signal (1:4 synchronism).

fibers) that code the incoming sound information (Kandel et al., 2013).

\section{H-reflex and reciprocal inhibition}

The H-reflex is a common electrical response used in clinical neurophysiology and in human research to estimate the excitability of the spinal cord (Daube and Rubin, 2009; Pierrot-Deseilligny and Burke, 2012). Basically, the H-reflex is generated by activating the monosynaptic pathway encompassing the Ia afferents, spinal MNs and the muscle. In this technique, the peripheral nerve is electrically stimulated by a rectangular pulse (1 ms duration), which evokes a synchronous discharge in the fibers. As Ia afferents have larger diameters than motor axons, a fraction of these fibers will respond to weak stimuli, generating a reflexive wave that can be recorded from the muscle EMG. Starting at some higher stimulus intensity, a few motor axons will also be activated generating a direct motor response (termed M-wave and occurring at a smaller latency) while the $\mathrm{H}$-reflex will attain its maximal amplitude. For stimulus intensities higher than this causing the maximum H-reflex, the antidromic APs generated in the motor axons will annihilate the orthodromic APs generated by the reflex. This will lead to a decrease in H-reflex amplitude. These mechanisms lead to a recruitment curve of the simulated responses as shown in Figure 7A, where the ordinate is the $\mathrm{H}$-reflex (or M-wave) normalized amplitude and the abscissa is the stimulus intensity. The data points in 
this graph were obtained using the neuromuscular model comprising the SOL muscle with stimulation of the PTN by single pulses of different amplitudes. The H-reflex and $\mathrm{M}$-wave recruitment curves resemble those obtained from human experiments (PierrotDeseilligny and Burke, 2012).

Frequently, the H-reflex of a given muscle (test reflex) is conditioned by a stimulus applied to a nerve innervating an antagonist muscle in order to evaluate the influence of the reciprocal inhibition (RI) pathway (Pierrot-Deseilligny and Burke, 2012). Using the simulator, one can assess the conditioning effect of the RI on the H-reflex by applying a test stimulus to the PTN and a conditioning stimulus to the CPN (antagonist nerve; see Figure 1) 2-3 ms before the test. Gray curves in Figure 7B represent
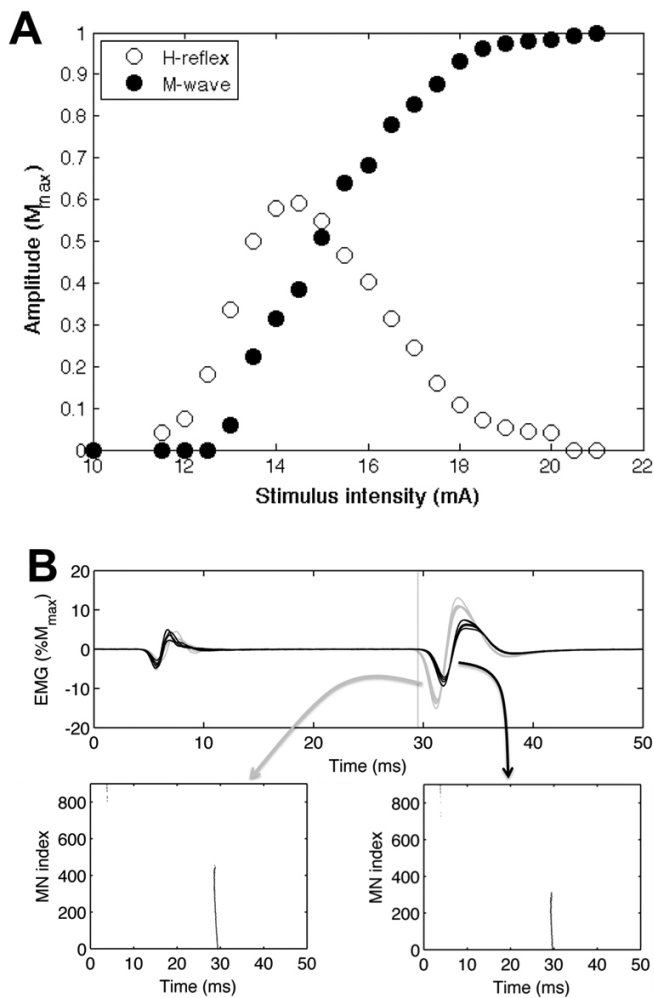

Figure 7. (A) H-reflex recruitment curve. Black circles represent the peak-to-peak amplitude of the direct motor M-wave, whereas empty circles correspond to peak-to-peak amplitude (in percentage of the maximum M-wave) of the H-reflex. (B) Effect of reciprocal inhibition (RI) on H-reflex amplitude. Top panel shows simulated EMGs $(n=5)$ evidencing small M-waves and the H-reflexes. Gray curves represent control conditions in which the RI pathway was not activated. Black curves show that the H-reflexes were reduced due to the conditioned stimulus applied to the Common Peroneal Nerve (CPN). Bottom panel shows two graphs with raster plots for one control and one RI condition (a raster plot shows for any $\mathrm{MN}$ of the pool, numbered in the ordinate, when it discharges in the time axis, as indicated by a single dot). Note the lower number of recruited MNs in RI condition. five sweeps of test $\mathrm{H}$ reflexes (simulated SOL muscle EMG) with amplitudes of $20 \%$ of the maximum M-wave $\left(\mathrm{M}_{\mathrm{MAX}}\right)$. When the conditioning stimulus is applied to the CPN, the H-reflex amplitude decreases by $\sim 40 \%$ of the control value (see black curves in Figure 7B). This amount of inhibition is compatible with experimentally observed values for humans (Morita et al., 1998). The lower panels in Figure 7B show the raster plots of active MNs for a single trial in control (left graph) and RI (right graph) conditions (in this case less MNs fired due to the reciprocal inhibition). This last piece of information is quite valuable (and unavailable from human experiments) to illustrate what happens to the neuronal population during studies of spinal reflexes.

\section{Recruitment and rate coding in force generation}

During a given motor task, two fundamental mechanisms are involved to increase the force level (Heckman and Enoka, 2012): i) the recruitment of new motor units, and ii) previously recruited motor units increase their firing rate, called rate coding in motor control. Both mechanisms may be easily studied using the neuromuscular simulator presented in this work.

Figure 8 shows the effects of a linearly rising command (1500 ms duration) imposed on the GM muscle and the resulting force increase in the upper panel. The shadowed box represents the recruitment range, i.e., the force range when additional (higher threshold) motor units are being recruited as the contraction increases. After the end of the recruitment range, the increase in force is due solely to the increase in motor unit firing rate. It is worth noting that for this muscle new motor units are recruited up to $\sim 80 \%$

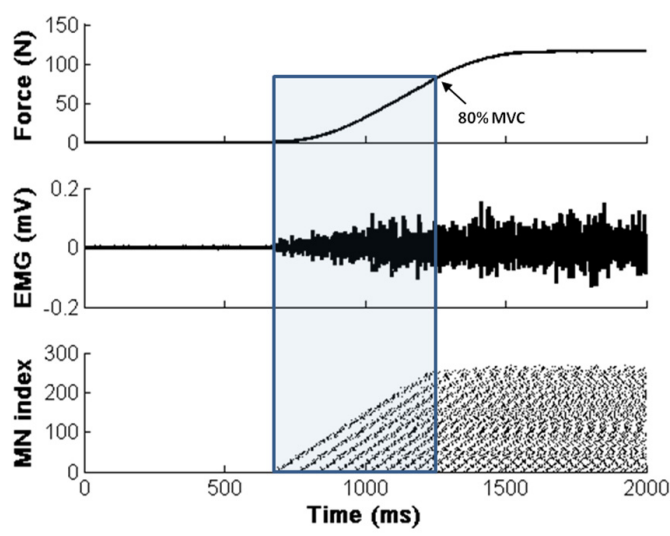

Figure 8. Recruitment and rate coding in force control. During a slowly rising contraction, new motor units are recruited up to $\sim 80 \%$ of the maximum voluntary contraction (MVC). Gray box defines the recruitment range in this case. The increase in muscle force observed above the full motor unit recruitment (to the right of the box) is due solely to the increase in motor unit firing rate (rate coding). 
of the maximum "voluntary" contraction (MVC). The graph in the middle panel of Figure 8 shows the simulated EMG. Note the increase in EMG intensity as the contraction progresses.

\section{Student feedback}

A survey was conducted on a subset of eight graduate students that took the Principles of Neuroscience course in 2012 at the USP. The questionnaire (Table 1) asked them about their opinion about ReMoto used in the second optional homework which explored several concepts, including H-reflex and force control (similar to the examples presented above). Identification field was not provided to avoid bias in the responses.

Figure 9 shows the survey outcomes. Almost all students rated the simulator as a good or an excellent learning tool, even when comparing with other simulators frequently used. They considered the graphical user interface adequate to be used by any student, but some (3) recommended a tutorial on the use of the simulator or onscreen tips so as to help the user in configuring a simulation. It should be noted that all the surveyed students completed successfully the proposed homework. All the students recommended the use of ReMoto as a learning tool for other students, and approximately $90 \%$ wrote that they would use the simulator in future projects.

\section{Discussion}

In this paper, we presented an open-source web-based neuromuscular simulator, ReMoto, which may be a helpful aid to the teaching of principles of neuroscience. The multi-scale structure of the simulator allows one to explore concepts ranging from single cell dynamics to whole muscle control, generating force and EMG. Due to its GUI, simulation configuration and data visualization are more straightforward than the previous version reported elsewhere (Cisi and Kohn, 2008). Its availability as a web application is a distinguishing characteristic in comparison to other packages (e.g., (Bower and Beeman, 1998; Brette et al., 2007; Carnevale and Hines, 2006; Delp et al., 2007)), mainly when viewed from the didactic perspective.

One of the guiding lights for the construction of this software was its usage by persons without a background on mathematical modeling and/or programming. The advantage of this approach is that all the structure needed for the simulation of a given neurophysiological experiment is already implemented. The user should only select the appropriate elements encompassing

Table 1. Questionnaire used in the survey. Questions were separated in two blocks: In block A the students rated the simulator as Excellent, Good, Irrelevant, Bad, and Poor; Block B comprised Yes/No questions.

\begin{tabular}{cl}
\hline Block(\#) & \multicolumn{1}{c}{ Question } \\
\hline $\mathrm{A}(1)$ & What is your opinion about the graphical user interface (GUI) of the simulator? \\
$\mathrm{A}(2)$ & What is your opinion about the ReMoto as a learning tool? \\
$\mathrm{A}(3)$ & How do you classify the ReMoto in comparison to other learning tools? \\
$\mathrm{A}(4)$ & What is your opinion about the way results are presented? \\
$\mathrm{B}(1)$ & Is it possible to use ReMoto with the information provided in the website? \\
$\mathrm{B}(2)$ & Do you recommend ReMoto to another student? \\
$\mathrm{B}(3)$ & Do you think you can use the ReMoto in the future? \\
$\mathrm{B}(4)$ &
\end{tabular}

A

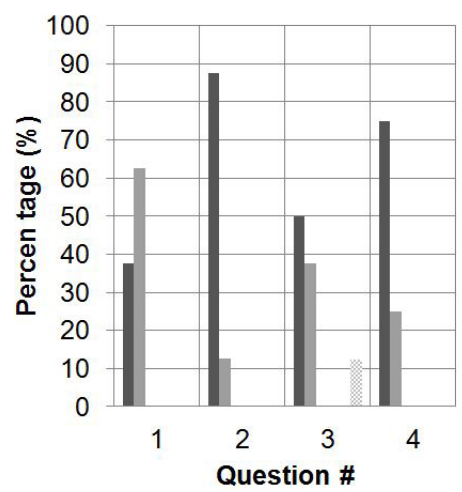

B

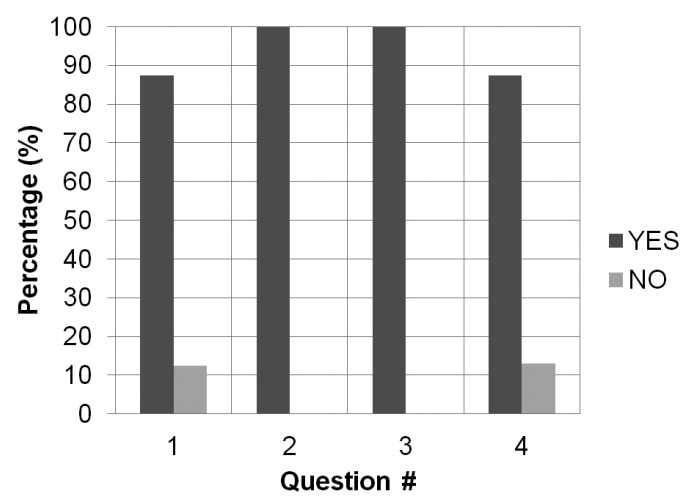

Figure 9. Survey outcomes. (A) Ratio for questions of the block A. (B) Ratio for questions of the block B. For questions, see Table 1. 
the neuronal circuitry it wants to simulate and then configure the inputs to the system. More advanced users can modify biophysical parameter values of each neuronal model (e.g., to study their influence on neuronal or motor behavior), or even download the source-code from the website in order to freely modify whatever he/she wants within the simulator. The object-oriented characteristic of the Java ${ }^{\circledR}$ programming language makes this task somewhat easy.

As mentioned earlier, the simulator has been used successfully as an additional teaching tool in a Principles of Neuroscience course at the USP. Due to its inherent power, the simulator could be used also in courses on Neural Engineering, Motor Control or Computational Neuroscience, as well as in research (Cisi and Kohn, 2008; Elias et al., 2012a). The instructor may use ReMoto in real time during classes to illustrate specific topics that are being taught (this has been done in previous years in the Principles of Neuroscience course mentioned before). At the same time, for the student, ReMoto may serve as an adjunct tool in the learning both at home or inside the classroom. It may act as a virtual laboratory in which the tutor (instructor) provides a challenging experiment and the student tries to figure out the best way to solve and interpret the problem using the knowledge acquired from the theory. In fact, several disciplines, including neurosciences, are being taught using a problem-based (or hands-on) approach (Deslauriers et al., 2011; Hosseinzadeh and Hesamzadeh, 2012; Wiznia et al., 2012). Technologies such as that presented here are in accordance with this teaching/learning method and may be used to improve education in neuroscience, neural engineering and related fields.

The outcomes of the survey conducted on graduate students from the Principles of Neuroscience course suggest that ReMoto was an effective resource. The (few) criticisms received are being used to improve future versions of the simulator, as well as in the development of additional documentation and video tutorials. A detailed quantitative evaluation of the efficacy of ReMoto as a learning tool may be a topic for a future study. Nonetheless, this preliminary result from a relatively small number of students was relevant to highlight the strengths and weaknesses of the simulator and was useful as a guide for future improvements.

The examples shown here (see Application examples in Results section) are just a small fraction of a multitude of possible scenarios that can be simulated in ReMoto. Several other examples frequently covered in principles of neuroscience courses can be also evaluated, for instance: $i$ ) drug effects on ionic channels (e.g., blocking) and their influence on AP firing threshold, or AP time-course; ii) effect of training on force generation, by changing the properties (i.e. amplitude and time-decay) of twitches; and iii) effect(s) of channelopathy (Cannon, 2006; Tomlinson et al., 2009; Vincent, 2010) on motor behavior. Just by changing parameters and/or adding elements one can simulate a whole new system that can be used to illustrate key concepts in neuroscience, neural engineering and motor control.

Some of the limitations of the simulator should be acknowledged: $i$ ) inexistence of a large dendritic tree, which is important to represent the spatial location of dendritic ionic channels (ElBasiouny et al., 2005) as well as synaptic dispersion (Segev et al., 1990); ii) the muscle force has no influence from intrinsic viscoelastic properties (Zajac, 1989); and iii) the simulator operates in open-loop, i.e., there is no proprioceptive feedback from peripheral receptors. Nevertheless, the system is being continuously incremented, and several new features are under way. A recent study from our lab (Chaud et al., 2012) used a beta version (not yet available as a webapplication) to evaluate the short-latency component of the stretch reflex of the SO muscle (Cronin et al., 2008). This new system included: muscle spindle model (Mileusnic et al., 2006) providing the proprioceptive feedback and a Hill-type muscle model (Zajac, 1989), with viscoelastic properties of muscle fibers and tendon. In the future, several of these new elements will be made available on the web application (as part of the ReMoto simulator) and several new examples may emerge for the teaching of fundamental mechanisms regarding the neuromusculoskeletal system.

\section{Acknowledgements}

This study was supported by grants from FAPESP and CNPq (Brazilian Funding Agencies). LAE holds a scholarship from FAPESP (proc. no. 2009/158020 ). The authors are grateful to V. M. Chaud for his invaluable work in improving the simulator's graphical user interface (GUI).

\section{References}

Bashor DP. A large-scale model of some spinal reflex circuits. Biological Cybernetics. 1998; 78(2):147-57. PMid:9525039. http://dx.doi.org/10.1007/s004220050421

Bower JM, Beeman D. The book of Genesis: Exploring realistic neural models with the general neural simulation system. New York: Springer; 1998.

Brette R, Rudolph M, Carnevale T, Hines M, Beeman D, Bower JM, Diesmann M, Morrison A, Goodman PH, Harris FC, Zirpe M, Natschlager T, Pecevski D, Ermentrout 
B, Djurfeldt M, Lansner A, Rochel O, Vieville T, Muller E, Davison AP, El Boustani S, Destexhe A. Simulation of networks of spiking neurons: A review of tools and strategies. Journal of Computational Neuroscience. 2007; 23(3):349-98. PMid:17629781 PMCid:2638500. http://dx.doi.org/10.1007/ s10827-007-0038-6

Cannon SC. Pathomechanisms in channelopathies of skeletal muscle and brain. Annual Review of Neuroscience. 2006; 29: 387-415. PMid:16776591. http:// dx.doi.org/10.1146/annurev.neuro.29.051605.112815

Carnevale NT, Hines ML. The Neuron book. New York: Cambridge University Press; 2006. http://dx.doi.org/10.1017/ CBO9780511541612

Chaud VM, Elias LA, Watanabe RN, Kohn AF. A simulation study of the effects of activation-dependent muscle stiffness on proprioceptive feedback and short-latency reflex. In: 4th IEEE RAS/EMBS International Conference on Biomedical Robotics and Biomechatronics: Proceedings of the International Conference on Biomedical Robotics and Biomechatronics; 2012; Rome. Rome: IEEE; 2012. p. 133-8.

Cisi RRL, Kohn AF. Simulation system of spinal cord motor nuclei and associated nerves and muscles, in a Web-based architecture. Journal of Computational Neuroscience. 2008; 25(3):520-42. PMid:18506610. http:// dx.doi.org/10.1007/s10827-008-0092-8

Cronin NJ, Peltonen J, Ishikawa M, Komi PV, Avela J, Sinkjaer T, Voigt M. Effects of contraction intensity on muscle fascicle and stretch reflex behavior in the human triceps surae. Journal Applied Physiology. 2008; 105(1):226-32. PMid:18483163. http://dx.doi.org/10.1152/japplphysiol.90432.2008

Daube JR, Rubin DI. Clinical Neurophysiology. 3rd. Oxford: Oxford University Press; 2009.

Deger M, Helias M, Boucsein C, Rotter S. Statistical properties of superimposed stationary spike trains. Journal of Computational Neuroscience. 2012; 32(3):443-63. PMid:21964584 PMCid:3343236. http://dx.doi.org/10.1007/ s10827-011-0362-8

Delp SL, Anderson FC, Arnold AS, Loan P, Habib A, John CT, Guendelman E, Thelen DG. OpenSim: open-source software to create and analyze dynamic Simulations of movement. IEEE Transactions on Biomedical Engineering. 2007; 54(11):1940-50. PMid:18018689. http:// dx.doi.org/10.1109/TBME.2007.901024

Deslauriers L, Schelew E, Wieman C. Improved learning in a large-enrollment physics class. Science. 2011; 332(6031):8624. PMid:21566198. http://dx.doi.org/10.1126/science.1201783

Destexhe A. Conductance-based integrate-and-fire models. Neural Computation. 1997; 9(3):503-14. PMid:9097470. http://dx.doi.org/10.1162/neco.1997.9.3.503

Destexhe A, Mainen ZF, Sejnowski TJ. An efficient method for computing synaptic conductances based on a kinetic-model of receptor-binding. Neural Computation. 1994; 6(1):14-8. http://dx.doi.org/10.1162/neco.1994.6.1.14

Dolecek GJ. MATLAB-Based Program for teaching autocorrelation function and noise concepts. IEEE Transactions on Education. 2012; 55(3):349-56. http:// dx.doi.org/10.1109/TE.2011.2176736
ElBasiouny SM, Bennett DJ, Mushahwar VK. Simulation of dendritic $\mathrm{Ca}(\mathrm{V}) 1.3$ channels in cat lumbar motoneurons: Spatial distribution. Journal of Neurophysiology. 2005; 94(6):3961-74. PMid:16120667. http://dx.doi.org/10.1152/jn.00391.2005

Elias LA, Chaud VM, Kohn AF. Models of passive and active dendrite motoneuron pools and their differences in muscle force control. Journal of Computational Neuroscience. 2012a; 33(3):515-31. PMid:22562305. http://dx.doi.org/10.1007/s10827-012-0398-4

Elias LA, Chaud VM, Watanabe RN, Kohn AF. A web-based neuromuscular simulator applied to the teaching of the basics of neuroscience. In: 42nd Annual Meeting of the Society for Neuroscience: Proceedings of the Annual Meeting of the Society for Neuroscience; 2012; New Orleans. New Orleans; 2012b. p. 23.06SA.

Elias LA, Kohn AF. Individual and collective properties of computationally efficient motoneuron models of types $\mathrm{S}$ and F with active dendrites. Neurocomputing. 2013; 99: 521-33. http://dx.doi.org/10.1016/j.neucom.2012.06.038

Fuglevand AJ, Winter DA, Patla AE. Models of recruitment and rate coding organization in motor-unit pools. Journal of Neurophysiology. 1993; 70(6):2470-88. PMid:8120594.

Heckman CJ, Enoka RM. Motor unit. Comprehensive Physiology. 2012; 2:2629-82. PMid:23720261.

Heckman CJ, Mottram C, Quinlan K, Theiss R, Schuster J. Motoneuron excitability: The importance of neuromodulatory inputs. Clinical Neurophysiology. 2009; 120(12):204054. PMid:19783207. http://dx.doi.org/10.1016/j. clinph.2009.08.009

Hodgkin AL, Huxley AF. A quantitative description of membrane current and its application to conduction and excitation in nerve. Journal of PhysiologyLondon. 1952; 117(4):500-44.

Hosseinzadeh N, Hesamzadeh MR. Application of project-based learning (PBL) to the teaching of electrical power systems engineering. IEEE Transactions on Education. 2012; 55(4):495-501. http://dx.doi.org/10.1109/ TE.2012.2191588

Illert M, Wiese H, Wolfram U. Electroencephalography and evoked potentials: a PC-based analysis program for laboratory courses in physiology. Advances in Physiology Education. 1992; 263(6 Pt 3):S16-22.

Kandel ER, Schwartz JH, Jessell TM, Siegelbaum SA, Hudspeth AJ. Principles of neural science. 5th ed. New York: McGraw-Hill; 2013.

Kernell D. The motoneuron and its muscle fibres. New York: Oxford University Press; 2006. PMid:17054451. http:// dx.doi.org/10.1093/acprof:oso/9780198526551.001.0001

Kohn AF, Floeter MK, Hallett M. Presynaptic inhibition compared with homosynaptic depression as an explanation for soleus H-reflex depression in humans. Experimental Brain Research. 1997; 116(2):375-80. http://dx.doi.org/10.1007/ PL00005765

Lo Conte LR, Merletti R, Sandri GV. Hermite expansions of compact support waveforms: applications to 
myoelectric signals. IEEE Transactions on Biomedical Engineering. 1994; 41(12):1147-59. PMid:7851916. http:// dx.doi.org/10.1109/10.335863

Martinez OD, Kohn AF, Segundo JP. Pervasive locking, saturation, asymmetric rate sensitivity and doublevaluedness in crayfish stretch receptors. Biological Cybernetics. 1983; 49(1):33-43. PMid:6652140. http:// dx.doi.org/10.1007/BF00336926

Maw S, Frank J, Greig G. A spinal circuitry simulator as a teaching tool for neuromuscular physiology. American Journal of Physiology. 1996; 270(6 Pt 3):S50-68. PMid:8712255.

McGrath P, Kucera R, Smith W. Computer simulation of introductory neurophysiology. Advances in Physiology Education. 2003; 27(3):120-9. PMid:12928321. http:// dx.doi.org/10.1152/advan.00055.2002

Mileusnic MP, Brown IE, Lan N, Loeb GE. Mathematical models of proprioceptors. I. Control and transduction in the muscle spindle. Journal of Neurophysiology. 2006; 96(4):1772-88. PMid:16672301. http://dx.doi.org/10.1152/jn.00868.2005

Milner-Brown HS, Stein RB, Yemm R. The contractile properties of human motor units during voluntary isometric contractions. Journal of Physiology. 1973; 228(2):285-306. PMid:4687100 PMCid:1331298.

Morita H, Petersen N, Christensen LO, Sinkjaer T, Nielsen J. Sensitivity of H-reflexes and stretch reflexes to presynaptic inhibition in humans. Journal of Neurophysiology. 1998; 80(2):610-20. PMid:9705454.

Morse TM. Neuroinformatics: from bioinformatics to databasing the brain. Bioinformatics and Biology Insights. 2008; 2:253-64. PMid:19812780 PMCid:2735961.

Pierrot-Deseilligny E, Burke D. The circuitry of the human spinal cord: Spinal and corticospinal mechanisms of movement. Cambridge: Cambridge University Press; 2012. http://dx.doi.org/10.1017/CBO9781139026727

Raphael G, Tsianos GA, Loeb GE. Spinal-like regulator facilitates control of a two-degree-of-freedom wrist. Journal of Neuroscience. 2010; 30(28):9431-44. PMid:20631172.

Segev I, Fleshman JW, Burke RE. Computersimulation of group Ia EPSPs using morphologically realistic models of cat alpha-motoneurons. Journal of Neurophysiology. 1990; 64(2):648-60. PMid:2213137.

Tomlinson SE, Hanna MG, Kullmann DM, Tan SV, Burke D. Clinical neurophysiology of the episodic ataxias: insights into ion channel dysfunction in vivo. Clinical Neurophysiology. 2009; 120(10):1768-76. PMid:19734086. http://dx.doi.org/10.1016/j.clinph.2009.07.003

Touretzky DS, Ladsariya A, Albert MV, Johnson JW, Daw ND. HHsim: an open source, real-time, graphical HodgkinHuxley simulator. In: 33rd Annual Meeting of the Society for Neuroscience: Proceedings of the Annual Meeting of the Society for Neuroscience; 2003; New Orleans. New Orleans; 2003. p. 29:24.13.

Vincent A. Autoimmune channelopathies: well-established and emerging immunotherapy-responsive diseases of the peripheral and central nervous systems. Journal of Clinical Immunology. 2010; 30 Suppl 1:S97-102. PMid:20387102. http://dx.doi.org/10.1007/s10875-010-9401-x

Winters JM. An improved muscle-reflex actuator for use in large-scale neuromusculoskeletal models. Annals of Biomedical Engineering. 1995; 23(4):359-74. PMid:7486344. http://dx.doi.org/10.1007/BF02584437

Wiznia D, Korom R, Marzuk P, Safdieh J, Grafstein B. PBL 2.0: enhancing problem-based learning through increased student participation. Medical Education Online. 2012; 17: 17375. PMid:22778540 PMCid:3374499. http://dx.doi.org/10.3402/meo.v17i0.17375

Zajac FE. Muscle and tendon: Properties, models, scaling, and application to biomechanics and motor control. Critical Reviews in Biomedical Engineering. 1989; 17(4):359-411. PMid:2676342.

Zhou P, Rymer WZ. MUAP number estimates in surface EMG: Template-matching methods and their performance boundaries. Annals of Biomedical Engineering. 2004; 32(7):1007-15. http://dx.doi.org/10.1023/ B:ABME.0000032463.26331.b3

Ziv I, Baxter DA and Byrne JH. Simulator for neural networks and action potentials: description and application. Journal of Neurophysiology. 1994; 71(1):294-308. PMid:7512628.

\section{Authors}

